\title{
IFE Chamber Technology - Status and Future Challenges
}

W.R. Meier, A.R. Raffrary, S. Abdel-Khalik, G. Kulcinski, J.F. Latkowski, F. Najmabadi, C.L. Olson, P.F. Peterson, A. Ying, M. Yoda

This article was submitted to

American Nuclear Society $15^{\text {th }}$ Fusion Topical Meeting, Washington, D.C. November $17-21,2002$

Law mence

Livermore

National

Laboratory

\section{November 15, 2002}




\section{DISCLAIMER}

This document was prepared as an account of work sponsored by an agency of the United States Government. Neither the United States Government nor the University of California nor any of their employees, makes any warranty, express or implied, or assumes any legal liability or responsibility for the accuracy, completeness, or usefulness of any information, apparatus, product, or process disclosed, or represents that its use would not infringe privately owned rights. Reference herein to any specific commercial product, process, or service by trade name, trademark, manufacturer, or otherwise, does not necessarily constitute or imply its endorsement, recommendation, or favoring by the United States Government or the University of California. The views and opinions of authors expressed herein do not necessarily state or reflect those of the United States Government or the University of California, and shall not be used for advertising or product endorsement purposes.

This is a preprint of a paper intended for publication in a journal or proceedings. Since changes may be made before publication, this preprint is made available with the understanding that it will not be cited or reproduced without the permission of the author.

This report has been reproduced directly from the best available copy.

Available electronically at http://www.doc.gov/bridge

Available for a processing fee to U.S. Department of Energy

And its contractors in paper from

U.S. Department of Energy

Office of Scientific and Technical Information

P.O. Box 62

Oak Ridge, TN 37831-0062

Telephone: (865) 576-8401

Facsimile: (865) 576-5728

E-mail: reports@adonis.osti.gov

Available for the sale to the public from

U.S. Department of Commerce

National Technical Information Service

5285 Port Royal Road

Springfield, VA 22161

Telephone: (800) 553-6847

Facsimile: (703) 605-6900

E-mail: orders@ntis.fedworld.gov

Online ordering: http://www.ntis.gov/ordering.htm

\section{OR}

Lawrence Livermore National Laboratory

Technical Information Department's Digital Library

http://www.llnl.gov/tid/Library.html 


\title{
IFE CHAMBER TECHNOLOGY - STATUS AND FUTURE CHALLENGES
}

\author{
W.R. Meier', A.R. Raffrary ${ }^{2}$, S. Abdel-Khalik ${ }^{3}$, G. Kulcinski ${ }^{4}$, J.F. Latkowski ${ }^{1}$,

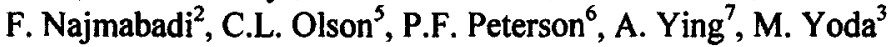 \\ 1. Lawrence Livermore National Laboratory \\ P.O Box 808, L-641, Livermore, CA 94551 \\ meier5@llnl.gov (925) 422-8536 \\ 2. University of California, San Diego, CA \\ 3. Georgia Institute of Technology, Atlanta, GA \\ 4. University of Wisconsin, Madison, WI \\ 5. Sandia National Laboratory, Albuquerque, NM \\ 6. University of California, Berkeley, CA \\ 7. University of California, Los Angeles, CA
}

\begin{abstract}
Significant progress has been made on addressing critical issues for inertial fusion energy (IFE) chambers for heavy-ion, laser and Z-pinch drivers. A variety of chamber concepts are being investigated including drywall (currently favored for laser IFE), wetted-wall (applicable to both laser and ion drivers), and thickliquid-wall (favored by heavy ion and z-pinch drivers). Recent progress and remaining challenges in developing IFE chambers are reviewed.
\end{abstract}

\section{INTRODUCTION}

Progress continues to be made on the science and technology needed for the development of inertial fusion energy (IFE) chambers. IFE chambers can be broadly classified into three categories: dry-wall, wetted-wall, and thick-liquid-wall. In dry-wall chambers, a low-density gas, armor coating or other engineered surface, possibly combined with a magnetic field, protects the first wall (FW) structures from short range $x$-rays and target debris, (generally about $30 \%$ of the fusion yield from an IFE capsule). The energy split between $\mathrm{x}$ rays and debris depends on the target design. (Debris, as used here, includes fusion alphas and other burn ions, unburned $D$ and $T$ ions, and energetic material from other target components such as the fuel capsule.) The most recent example of an integrated dry-wall chamber design is Sombrero [1,2]. Dry-wall chambers are designed with robust target-facing materials to avoid surface erosion and maintain very clean chamber conditions.
Wetted-wall chambers use a thin liquid film or spray to directly absorb $x$-rays and debris. A large fraction of the $x$-ray and debris energy is removed by vaporization of the liquid, combined with sensible heating of the liquid to higher temperature if the liquid layer is designed to have significant flow rates. Heat removal by vaporization or sensible heating of a liquid layer reduces the importance of conduction through a solid first wall to a flow coolant, which reduces the mass required for the first-wall structure as well as its sensitivity to neutron induced swelling and strength reduction. Recent wetted-wall examples include Prometheus, Osiris and Koyo [2-4].

Thick-liquid-wall chambers, such as HYLIFE-II, provide a neutronically thick layer of liquid between the target and structures, thus providing protection not only from the shot-range target emissions buy also neutrons [5]. Due to the small target-facing surface area in thick-liquid IFE chambers, $x$-ray and debris energy are removed primarily by the vaporization from the inner pocket surface, followed by recondensation on droplet sprays in other regions of the chamber.

Many analogous issues exist for magnetic fusion energy (MFE) chambers, and current MFE chamber research is also investigating dry and liquid protected blanket systems [6]. In this paper, we describe the status of R\&D for IFE chambers, highlight recent experimental and modeling results, and note synergies with MFE chamber research and remaining challenges. 


\section{DRY-WALL CHAMBERS}

\section{II.1 Background}

Several examples of early dry-wall chambers are reviewed in Ref. 7. In the early 1990's, the Sombrero chamber concept was developed [1,2], and a modification called SIRIUS was also published [8]. Sombrero, illustrated in Fig. 1, has served as the starting point for more recent studies of dry-wall chambers by the ARIES team [9] and the chamber R\&D carried out by the High Average Power Laser (HAPL) program [10]. The original Sombrero design used a 3D carbon/carbon composite for the first wall and integrated blanket structure. The $6.5-\mathrm{m}$ radius chamber was filled with 0.5 torr of $\mathrm{Xe}$ gas. The $\mathrm{Xe}$ gas absorbs the $\mathrm{x}$ rays and debris and forms a fireball which reradiates over a longer time reducing the peak power incident on the FW. Sublimation was thus avoided.

The pulsed heating of dry-wall chamber surfaces has some analogy to the disruption heating of MFE tokamak chambers, so some synergies exist in the development of plasma-facing armor systems for debris and x-ray heat removal. The requirements for the blanket structures below the armor, which remove heat deposited by neutrons and provide for tritium breeding, are closely analogous to requirements for MFE dry-blanket systems. Key issues thus center on armor design for the pulsed IFE heating environment.

\section{II.2 Key Issues for Dry-Wall Chambers}

Key issues for dry-wall chambers include:

1) Protecting the FW from target $x$ rays and debris or developing a material that can survive these threats. The first wall must survive both single-shot (i.e., avoid vaporization and melting) and long-term effects (e.g., cyclic fatigue).

2) Neutron damage lifetime of the first wall and blanket structures. The first wall must survive neutron damage effects for a reasonably long time $(>1-5$ years), maintaining its functional capabilities (structural, heat conduction, etc.). It must also be designed for rapid replacement or maintenance to minimize the impact on plant availability.

3) Re-establishing pre-shot chamber conditions between pulses. For example, the gas fill, if used, must return to the density conditions needed to serve its protective function.

4) Interface issues, including a) effects of chamber conditions on the survival and trajectory of the injected cryogenic target and b) beam propagation,

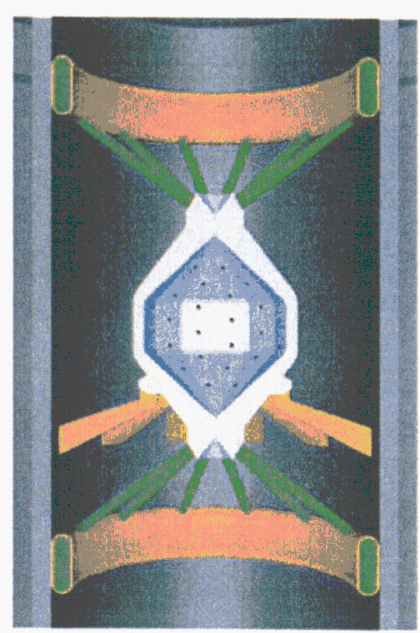

Fig. 1. Sombrero is an example of a dry-wall chamber.

are also important. These are of particular concern for the gas filled approach.

\section{II.3 Dry-Wall R\&D}

Dry-wall chamber research has benefited from ARIES work on IFE over the past couple years [9] and from the HAPL program which is focused on the dry-wall approach $[10,11]$. The IFE chamber wall requirements of integrity, lifetime and compatibility with reactor operation are quite demanding in view of the challenging cyclic operating conditions both in terms of incident heat and particle fluxes.

Recent work by the HAPL chamber design team has focused on armor coating materials for the first wall. Candidate armor materials must have high temperature capability and good thermal properties for accommodating energy deposition and providing the required lifetime. Carbon and refractory metals (e.g. tungsten) have reasonably high thermal conductivity at high temperature $(\sim 100-200 \mathrm{~W} / \mathrm{m}-\mathrm{K})$ and high phase change temperature (sublimation temperature of carbon $\sim 3370^{\circ} \mathrm{C}$; melting point of tungsten $\sim 3410^{\circ} \mathrm{C}$ ) and are considered as candidate materials.

The thermal response of the first wall is being modeled for various chamber conditions (gas or no gas), targets, and materials. Figure 2 shows an example of the temperature history in a $\mathrm{W}$ armor when subjected to the output of a $154 \mathrm{MJ}$ direct-drive target. The chamber radius is $6.5 \mathrm{~m}$, and there is no buffer gas. In this case, the peak temperature remains below the melting point of $\mathrm{W}$. Also note that only the inner most region $(\sim 10$ 's $\mu \mathrm{m})$ experiences the severe temperature excursion. 


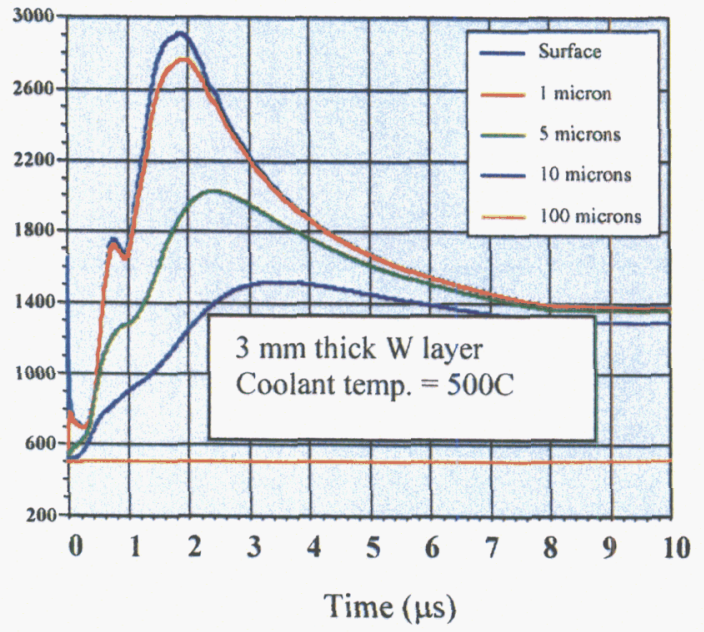

Fig. 2 Tungsten armor temperature (C) versus time. (6.5 $\mathrm{m}$ radius chamber, no gas fill, $154 \mathrm{MJ}$ target)

In addition, the possibility of utilizing an engineered surface (such as a high porosity carbon fibrous carpet) to maximize the effective incident area and provide better accommodation of high-energy deposition is being considered. Figure 3 shows an example of a carbon fiber carpet developed by ESLI. This material was exposed to $5 \mathrm{~J} / \mathrm{cm}^{2}$ x-ray pulse on Sandia's Z-machine and showed no visible damage. Other materials, such as $\mathrm{SiC}$, could also be made into such carpets. The Z-machine is also being used to test other candidate FW materials.

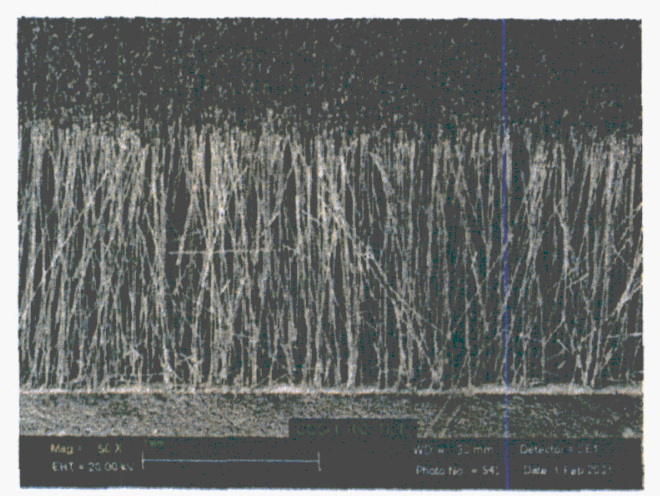

Fig. 3. Carbon "carpet" is a possible first wall material.

For carbon, a major concern for the design, operation, and safety of the system is the erosion of the carbon armor over many pulses, and distribution of eroded material in combination with tritium. Refractory metals, such as tungsten, provide high temperature capability without the major tritium inventory concern. However, melting is an issue for high-energy deposition and the stability of the melt layer and integrity of the resolidified material must be addressed. The effect of helium ion implantation on the armor integrity is also a key issue in particular for tungsten in which He diffusion is very slow. The HAPL program also includes investigation of protecting the first wall from ion damage using magnetic deflection, a concept originally proposed in the 1970's [12].

$R \& D$ effort is needed on the fabrication of the armor material, on its bonding to a structural material, and on the armor and bond integrity under high-heat-flux operation. In the case of IFE particular concerns exist as to the applicability of material (and bonding) properties and behavior evolved under equilibrium or moderate transients to the highly cyclic conditions at the armor surface. Even in the most optimistic case, it is difficult to guarantee that locally the armor will not erode to unacceptable level or fail. Thus, it is imperative that, in parallel with the R\&D effort, methods for in-situ repair of the armor be developed to avoid long and costly shutdown for replacement of major wall sections in the event of local failure or erosion

\section{II.4. Remaining Challenges - Dry Walls}

The various dry-wall approaches are still early in their development. Finding the best approach to dealing with short-range emission remains a key challenge. As indicted, a variety of ideas are being pursued with various levels of effort including gas-protected, armor-coated or other engineered surfaces, and magnetic deflection of debris. Developing a FW structural material with adequate neutron damage life is also a key challenge yet to be met. IFE will take advantage, to the extent possible, of radiation damage testing material development carried out by MFE. In addition, the IFE engineering test facility (ETF) will serve as an integrated neutron damage testing and material development facility for FWs and blankets. Since for IFE neutrons are emitted from essentially a point (the target), it is straightforward to scale the chamber dimensions to achieve a power-plant prototypic neutron wall loading (or higher for accelerated materials and component testing) with a low fusion power (100's of MW) in the ETF.

\section{WETTED-WALL CHAMBERS}

\section{III.1 Background}

Wetted-wall chambers, where a thin liquid film absorbs $\mathrm{x}$ rays and debris, date to the earliest days of IFE chamber development $[13,14]$. Several variations have been proposed with the most recent being the Prometheus (Fig. 4), Osiris and Koyo designs. The protective liquid layer is establish by several methods (or combinations), including injection of thin sheet flow, forced flow through 
porous materials (either rigid walls as in Prometheus or flexible walls as in Osiris), and sprays to provide the coating [15].

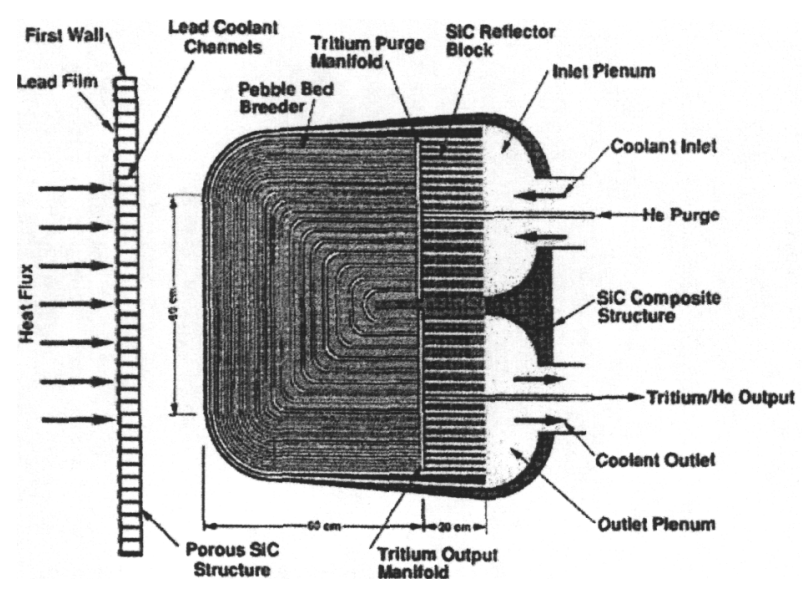

Fig. 4. Prometheus porous wetted wall and blanket.

\subsection{Key Issues for Wetted-Wall Chambers}

The key issues for wetted-wall chambers are similar to dry-walls and include:

1) Assuring that the protective layer can be established in the first place and reliably re-established in the inter-pulse time. Dry-out of any area must be avoided. Particularly important fluid mechanics issues are the maintenance of liquid films on non-wetting structural substrates, and control of dripping on downwardfacing and inclined surfaces.

2) Interface issues, primarily recovery of in-chamber conditions, by condensation or pumping, to return to a low enough the vapor density to allow beam and target propagation.

3) Neutron damage life of low-mass flow-guiding structures. Like the FW in a dry-wall chamber, the underlying flow-guiding structure of the wetted with receive a high $14 \mathrm{MeV}$ neutron wall loading. Since the FW in a wetted wall design does not necessarily have to conduct heat to the blanket, there is more design flexibility than with a dry wall, and thus it may be possible that higher radiation damage level can be tolerated. For example, flexible fabric weaves as in HIBALL, Osiris and Koyo, should be more tolerant to neutron induced swelling than rigid walls, but this remains to be demonstrated.

\section{Wetted Wall R\&D}

While significant modeling has been done, it is only recently that experiments on wetted walls have begun. Work at Georgia Tech on liquid sheets injected along a solid wall is reported in these proceedings [16]. They have also begun modeling of flow through porous structures to determine detachment time of drops from horizontal and inclined surfaces as a function of fundamental fluid and structure properties [17]. Also, the MFE APEX team has done significant work on injected liquid films [6].

Experimental and numerical studies as GT have been conducted to examine the fluid dynamic aspects of thin liquid film protection systems with either radial injection through a porous first wall or forced flow of a thin liquid film tangential to a solid first wall. For a wetted wall chamber, the downward facing surfaces are critical since they can be sources of drops that could interfere with beam or target injection. Typical results showing the evolution of the free surface for a liquid lead film on a horizontal downward-facing surface are shown in Fig. 5. The film is assumed to be at $700 \mathrm{~K}$ with an initial thickness of $1.0 \mathrm{~mm}$ and an injection velocity of $1.0 \mathrm{~mm} / \mathrm{s}$; a random initial perturbation with maximum amplitude of $1.0 \mathrm{~mm}$ is applied at the beginning of the transient. These results suggest that droplet detachment occurs nearly $0.38 \mathrm{~s}$ after the initial perturbation is imposed.
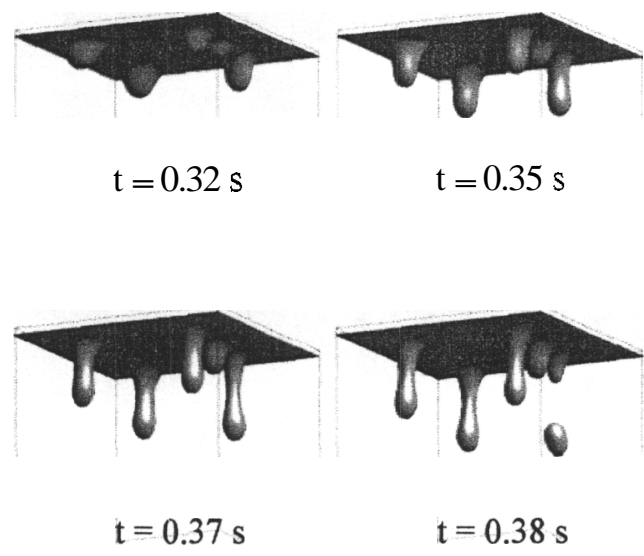

Fig. 5: Evolution of lead film surface on a downward facing first wall with random initial perturbation.

In addition to the fluid dynamics needed to establish the protective liquid layer, several other aspects of wetted wall chambers have been investigated by the recent ARIES IFE study [22]. These include

1) Condensation. The time required for film condensation to clear the chamber as a function of $\mathrm{Pb}$ and FLiBe vapor pressure and temperature has been calculated. Condensation kinetics does not appear to be a rep-rate limiting issue. 
2) Aerosol Formation and Behavior. Source terms for aerosol formation include ablation of the film due to the initial high-energy deposition and formation of aerosol due to in-flight condensation in saturated regions. Aerosol sizes and densities at different chamber locations prior to each shot have been estimated.

3) Design Windows. Acceptable operating windows in terms of vapor pressure and aerosol density to allow beam propagation and target injection have been studied. Results, of course, depend on the target type (direct or indirect drive) and driver (ion or laser beam).

\section{III.4 Remaining Challenges - Wetted Walls}

Like all concepts, wetted-walls are in an early stage of development. Engineering and testing of concepts for establishing the film in a lab setting have yet to be done (except for preliminary tests on injected films). Experiments and analyses to find the correct combination of porous structure, its composition, fluid pressure, geometry, etc. are needed, including the issues of fluid mechanics with wetting and non-wetting substrate materials. The next steps would then be to simulate fusion induced vaporization and disruption of the protective layer and study reestablishment issues. Nuclear testing of film and chamber response and FW material life will then be performed in the ETF.

\section{THICK-LIQUID-WALL CHAMBERS}

\section{IV.1 Background}

Thick-liquid wall (TLW) chambers also date to the early days of IFE R\&D (see examples in Ref. 7). The most recent version is HYLIFE-II, which uses oscillating jets of molten salt to dynamically clear the target region (Fig. 6). The TLW is currently the baseline approach for heavy ion

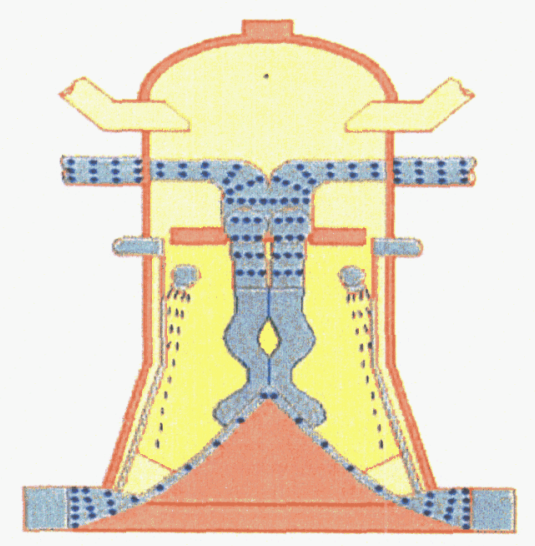

Fig. 6. HYLIFE-II thick liquid wall chamber. fusion with indirect drive targets and an updated HIF power plant is presented in Ref. 18. Z-pinch driven IFE also proposes to use a TLW chamber [19]. The great attraction of the TLW concept is that, if successful, the structural materials are protected by several neutron meanfree-paths of renewable liquid and will thus be able to survive much longer than unshielded walls, with virtually all structures lasting for the life of the plant. This may be possible with existing steels or near-term steel such at the low-activation ferritics being developed by the fusion community.

\section{IV.2 Key Issues for TLW Chambers}

Key issues for TLW chamber are:

1) Establishing the thick liquid blanket configuration and reestablishing it between pulses. There are many fluid dynamic issues, as discussed below, for the different types of jets needed in TLW chambers.

2) Assuring that beam and target inject paths are clear of drops of sufficient size or density to prevent injection and beam propagation, and stopping debris transport up beam tubes. (This is avoided with a Z-driver.)

3) Chemistry and material compatibility - issues related to the use of molten salt, effects of ionizing radiation and nuclear reactions (free fluorine), recovery of hohlraum materials, etc. Selection of a structural material that is compatible with the molten salt at the desired operating temperature $\left(\sim 600^{\circ} \mathrm{C}\right)$ and having good radiation damage tolerance is a key consideration.

\section{IV.3 R\&D on Thick Liquid Walls}

Over the past five years, significant progress has been made on various aspects of TLWs in multiple university experiments. Several universities are conducting smallscale experiments and modeling of the fluid dynamics of the various types of jets needed to form the protective liquid blanket. The University of California, Berkeley (UCB) has developed the type of oscillating jets needed to form the oscillating pocket. Their recent work (Fig. 7) has included demonstration of multiple disruptions and recovery of an oscillating 96-jet array using rapid chemical detonations [20].

UCB has demonstrated the generation of highly smooth and precise cylindrical liquid jets for use in forming grids for beam arrays [21]. Work on the interface between the accelerator and the chamber in the area of the final focus magnets has led to an innovative liquid vortex protection scheme. UCB has also recently experimentally demonstrated the performance of a new nozzle for creating a smooth liquid vortex within a circular tube, which can be 

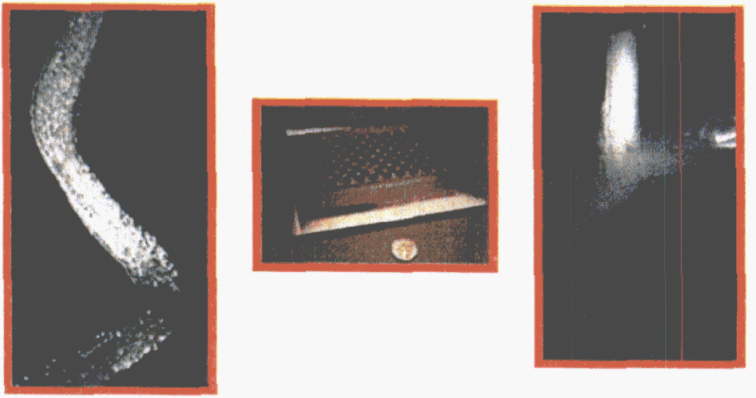

Fig. 7. Water jet experiments at UCB showing oscillating jet (left), 96-jet nozzle plate (center), and disruption of jet array using chemical detonation (right).

used in the bore of the final focus magnets to prevent direct exposure to $\mathrm{x}$-rays and debris and will also serve as a condensation region for hot vapor that flows up the beam lines. Recent studies have also investigated the addition of sodium fluoride to flibe, creating flinabe, to lower the melting temperature. This allows the molten salt to be used at temperatures below $400^{\circ} \mathrm{C}$ in the beam tubes, creating extremely low vapor pressure in this region.

The University of California, Los Angeles (UCLA) has recently built an experiment (Fig. 8.) to study the vaporization and condensation of flibe [22]. They are using a plasma gun to produce rapid vaporization (creating a few-eV plasma, the prototypical temperature expected in IFE chambers) and then diagnose vapor flow and condensation in a test chamber. The test chamber is being adapted to include liquid films and jets to simulate condensation in a HYLIFE-type chamber.

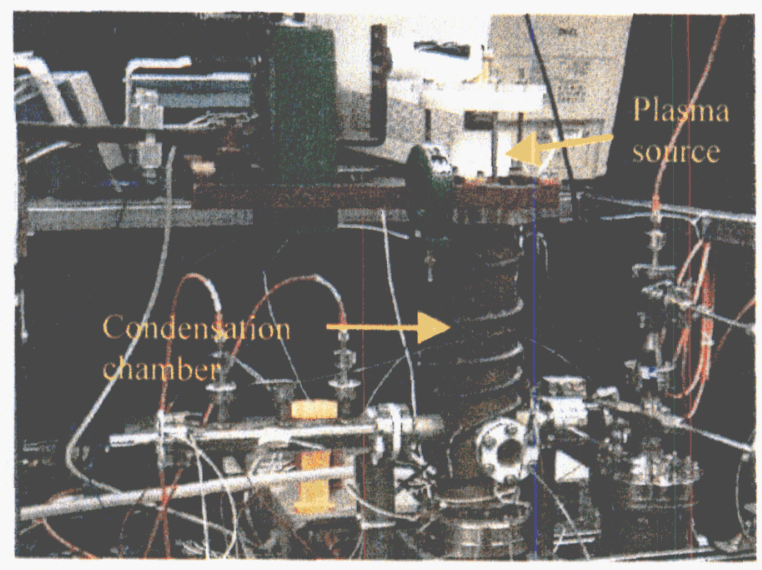

Fig. 8. Plasma gun based flibe condensation experiment at UCLA.
Georgia Institute of Technology (GT) has also done experiments on oscillating liquid jets using water. The current focus of their work is on characterization of the surface ripple on jets $[23,24]$. Figure 9 shows laserinduced fluorescence technique use to determine the surface quality of a water jet (slab shaped) dyed with fluorescein. Producing high quality jets with minimal surface ripple is important for the crossing jets that form the beam ports. The goal is to have these jets as close to the beams as possible (to provide good neutron shielding) while avoiding any clipping of the incoming beams by the jets. Results to date indicate that with proper nozzle design, the ripple on the order of a couple millimeters over the characteristic distance will be achievable. New work at GT will investigate mechanisms for drops being ejected from the surfaces of jets, which could be an issue if these drops interfere with target injection or beam propagation.

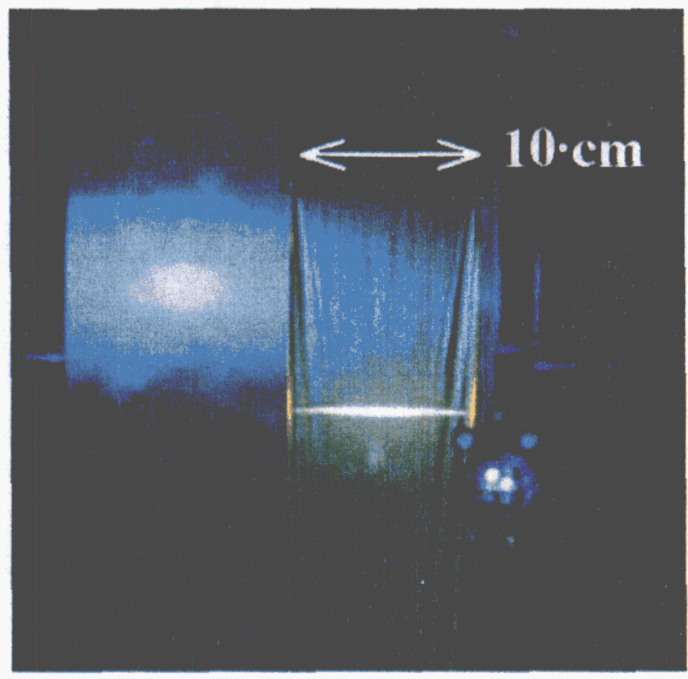

Fig. 9. Experiment at Georgia Tech to measure surface quality of a $1 \times 10 \mathrm{~cm}$ water jet.

\section{IV.4 Remaining Challenges - Thick Liquid Walls}

Remaining challenges include the need for additional of liquid jet experiments of increasing complexity to the point of scaled simulations of the full protective pocket and crossing jet geometry. Once established, disruption by chemical detonation to study rep-rate effects will be needed. A facility to study the chemical corrosion and erosion effects of high velocity molten salt jets, in particular for the sensitive nozzle structures, will be needed. Synergy with MFE studies will be useful here as well. Finally, as with other chamber concepts, nuclear effects testing, such as neutron induced isochoric heating and disruption of liquid structures, will be performed in the ETF. 


\section{CONCLUSIONS}

Progress on key issues for IFE chambers has occurred rapidly in the last several years. Experiments are being conducted on liquid protected wall concepts (using either thin films or injected jets) and on candidate materials for dry-wall chambers. Modeling of chamber phenomena (interaction of target emissions with in-chamber gases, liquids and structural materials) is also progressing. Many challenges remain, many related to the pulsed nature of IFE. There are also many opportunities for continued innovation and the design flexibility afforded by the separability of drivers, targets and chambers give us confidence that attractive solutions will be found.

\section{ACKOWLEDEMENTS}

The authors wish to acknowledge the contribution of many others at their respective home institutions for their contributions to the work on the aspects of IFE chambers reported here. Work of the LLNL authors performed under the auspices of the U.S. Department of Energy by the University of California, Lawrence Livermore National Laboratory under contract No. W-7405-ENG48.

\section{REFERENCES}

1. I.N. Sviatoslavsky et al., "A KrF Laser Driven Inertial Fusion Reactor SOMBRERO," Fusion Technol., 21 (N3), 1480-1474 (1992).

2. W.R. Meier, "Osiris and Sombrero Inertial Fusion Power Plant Designs - Summary, Conclusions, and Recommendations," Fusion Engin. and Design, 25, 145 (1994).

3. L.M. Waganer, "Innovation Leads the Way to Attractive Inertial Fusion Energy Reactors Prometheus-L and Prometheus-H," Fusion Engin. and Design, 25, 125 (1994

4. Kozaki, y et al, Proc. $7^{\text {th }}$ Int. Conf. On Emerging Nuclear Energy Systems, p 76 (1994).

5. R.W. Moir et al., "HYLIFE-II: A molten-salt inertial fusion energy power plant design-final report, Fusion Technol. 25 (1994) 5.

6. M. A. Abdou, and the APEX Team, Fus. Eng. \& Des., 54 (2001) 181.

7. M.J. Monsler et al., "An Overview of Inertial Fusion Reactor Design," Nuclear Technol./Fusion 1 (1981) 302.

8. I.N. Sviatoslavsky et al., "A Near Symmetrically Illuminated Direct Drive Laser Fusion Power Reactor - Sirius-P," Fusion Technol. 26, 868 (1994).

9. F. Najmabadi et al., "Assessment of Chamber Concepts for Inertial Fusion Energy Power Plants The ARIES-IFE Study," Proc Sym. on Inerital
Fusion Science and Technology (IFSA2001) Elsevier, p. 701 (2002).

10. See http://aries.ucsd.edu/HAPL/

11. R. Raffray, G. Federici, A. Hassanein and D. Haynes "Dynamic Chamber Armor Behavior in IFE and MFE," Presented at the 6th Intl. Symp. on Fusion Nuclear Technology, 7-12 April 2002, San Diego, California, USA. Submitted for publication in Fusion Engin. and Design.

12. L.A. Booth and T.G. Frank, "Commercial Applications of Inertial Confinement Fusion," LANL Rep. LA-6838-MS (May 1977).

13. L.A. Booth, "Central Station Power Generation by Laser-Driven Fusion," LANL Rep. LA-4858 (1972).

14. B. Badger et al., "HIBALL-II An Improved Conceptual Heavy Ion Beam Driven Reactor Study," Rep. UWFDM-625, Univ. of Wisconsin, Madison (1985).

15. UW spray design.

16. J.K. Anderson et al., "Experimental Studies of HighSpeed Liquid Films on Downward-Facing Surfaces," these proceedings.

17. S. Shin et al., "Effects of Surface Evaporation and Condensation on The Dynamics of Thin Liquid Films for The Porous Wetted Wall Protection Scheme In IFE Reactors," these proceedings

18. W.R. Meier, et al., "An Updated Design Point for Heavy Ion Fusion," these proceedings.

19. G.E. Rochau et al., "An IFE Power Plant Using ZPinch Indirect Drive," these proceedings.

20. S. Pemberton, C. Jantzen, J. Kuhn and P.F. Peterson, "Partial-pocket experiments for IFE thick-liquid pocket disruption and clearing," Fusion Technol. 39 (2001) 726.

21. R. Abbott, et al., Cylindrical liquid jet grids for beamport protection of thick-liquid heavy-ion fusion target chambers, Fusion Technol. 39 (2001) 732.

22. P. Claderoni, A. Ying, T. Sketchely and M. Abdou, "Description of a facility for vapor clearing rates studies of IFE reactors flibe liquid chambers," Fusion Technol. 39 (2001) 711 .

23. J. A. Collins, M. Yoda and S.I. Abdel-Khalik, "Direct measurements of free-surface smoothness in turbulent liquid sheets," Fusion Technol. 39 (2001) 721.

24. S. G. Durbin et al., "Turbulent Liquid Sheets for Protecting IFE Reactor Chamber First Walls, SG. Durbin," these proceedings. 